\title{
A Rectifying Acceptance Sampling Plan Based on the Process Capability Index
}

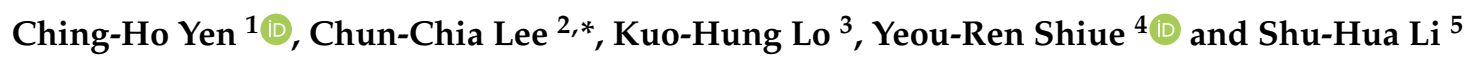 \\ 1 Department of Industrial Engineering \& Management Information, Huafan University, New Taipei City 223, \\ Taiwan; jimyen@cc.hfu.edu.tw \\ 2 School of Business, Minnan Normal University, Zhangzhou 363000, China \\ 3 Department of Economic \& Statistics, Fujian Business University, Fuzhou 350016, China; \\ kuohung30@fjcc.edu.cn \\ 4 Department of Management Engineering, Fujian Business University, Fuzhou 350016, China; \\ yrshiue@gmail.com \\ 5 Graduate Institute of Technology, Innovation and Intellectual Property Management, College of Commerce, \\ National Cheng-chi University, Taipei City 116, Taiwan; snowandbutterfly@gmail.com \\ * Correspondence: chunchia.derek@gmail.com; Tel.: +86-596-252-7183
}

Received: 21 December 2019; Accepted: 17 January 2020; Published: 20 January 2020

check for updates

\begin{abstract}
The acceptance sampling plan and process capability index (PCI) are critical decision tools for quality control. Recently, numerous research papers have examined the acceptance sampling plan in combination with the PCI. However, most of these papers have not considered the aspect of rectifying inspections. In this paper, we propose a quality cost model of repetitive sampling to develop a rectifying acceptance sampling plan based on the one-sided PCI. This proposed model minimizes the total quality cost (TQC) of sentencing one lot, including inspection cost, internal failure cost, and external failure cost. In addition, sensitivity analysis is conducted to investigate the behavior of relevant parameters against TQC. To demonstrate the advantages of the proposed methodology, a comparison is implemented with the existing rectifying sampling plan in terms of TQC and average outgoing quality limit. This comparison reveals that our proposed methodology exhibits superior performance.
\end{abstract}

Keywords: acceptance sampling plan; process capability indices; rectifying inspection; average outgoing quality limit; sensitivity analysis

\section{Introduction}

The acceptance sampling plan and process capability index (PCI) are critical for quality control in manufacturing. The acceptance sampling plan is used to determine the acceptance or rejection of one lot based on information obtained from a sample and is applied extensively in the inspection of raw materials, semifinished products, final products, and shipments. Acceptance sampling plan can be divided into two types, attributes and variables sampling plans. In comparison with attributes sampling plan, variables sampling plans provide a less sample size to attain the same protection for producer and purchaser as well as give more information about lots. Such saving of sample size may be especially marked if inspection is destructive and the item is expensive [1-3]. By contrast, the PCI establishes the relationship between the actual manufacturing process performance and manufacturing specifications, which quantifies the effectiveness of the production process. This process capability information measures the effectiveness of the production process. Since the use of the PCI and acceptance sampling plans became popular, numerous papers have combined the two to develop variables in sampling plans for lot sentencing [4-14]. These papers are designed for different purposes 
or sampling skills. Yen et al. [4,12], Aslam et al. [6,13], Lee et al. [9], and Wu and Wang [11] used PCI to develop variables sampling plans which consider the quality of ongoing lot and preceding lots for lot sentencing. Wu and Liu [5] designed a single variables sampling plan based on process yield index. Wu et al. [7] proposed a PCI-based sampling plan for resubmitted lots which examined the situation where resampling is permitted on lots not accepted on original inspection. Yen et al. [8] and Fallah-Nezhad and Seifi [10] used PCI to design repetitive group sampling (RGS) plans for determination of lot.

Research combining acceptance sampling plans and the PCI has seldom focused on rectifying inspection, where $100 \%$ inspection will be carried out if one lot is rejected. Rectifying inspection is frequently implemented because of supplier monopoly or urgent buyer demand. Rectifying inspection means that if one lot is accepted, then only defective units detected from $\mathrm{n}$ units are replaced with nondefective units. However, if the lot is rejected, $100 \%$ of the remaining units are inspected and all defective units are replaced with nondefective units. After this process is completed, the lot rejected can be accepted. Three quality costs are incurred in rectifying inspection: inspection cost, internal failure cost, and external failure cost. Inspection cost occurs when products are inspected. Internal failure cost occurs when products fail to meet the minimum quality requirements prior to delivery to consumers. External cost occurs when products fail to meet the minimum quality requirements after delivery to consumers. More detailed information regarding quality cost is presented in the study conducted by Feigenbaum [14], Juarn [15], and Shank and Govindarajan [16].

These statements in the previous paragraph imply that the application of rectifying inspection is a practical problem for lot sentencing. Yen et al. [17] used a single sampling scheme to develop a rectifying sampling plan based on a one-sided PCI. However, other flexible sampling schemes, such as repetitive sampling [18], multiple dependent state sampling [19], and multiple dependent state repetitive sampling [20], have been presented with smaller sample sizes than that of the single sampling scheme. Therefore, we develop a rectifying sampling plan that has a lower total quality cost (TQC) than that reported by Yen et al. [17]. An exploration of the relevant literature reveals that research on rectifying sampling plans with flexible sampling schemes, such as repetitive group sampling, multiple dependent state sampling, and multiple dependent state repetitive sampling have not yet been conducted. Thus, in this paper, we apply a repetitive sampling scheme to design a rectifying sampling plan based on the concept of Yen et al. [17], which is called a repetitive rectifying sampling plan. The remainder of this paper is organized as follows. PCIs are introduced in Section 2. The design of our proposed sampling plan is presented in Section 3. Parameters for the rectifying sampling plan and the results of the sensitivity analysis are provided in Section 4. Finally, this paper's conclusions are presented in Section 5.

\section{Process Capability Indices}

PCIs have been proposed to provide numerical measures of process performance in the manufacturing industry. These indices establish the relationship between the actual process performance and manufacturing specifications, and they have been a focus of recent research in statistical and quality assurance. The most popular capability indices, $C p$ [21], Cpk [22] and Cpm [23], are widely used in the manufacturing industry to evaluate process performance for cases with two-sided specification limits. The index $C p$ measures the overall process variation relative to specification tolerance. The index $C p k$ takes into account the magnitude of process variation as well as the degree of process centering. The index $\mathrm{Cpm}$ measures the ability of the process to cluster around the target, which reflects the degrees of the process target. The explicit forms of indices are defined as follows.

$C_{p}=\frac{U S L-L S L}{6 \sigma}, C_{p k}=\min \left\{C_{p u}, C_{p l}\right\}=\min \left\{\frac{U S L-\mu}{3 \sigma}, \frac{\mu-L S L}{3 \sigma}\right\}$, and $C_{p m}=\frac{U S L-L S L}{6 \sqrt{\sigma^{2}+(\mu-T)^{2}}}$,

where USL and LSL are the upper and lower specification limits, respectively, $T$ is the target value, $\mu$ is the process mean, and $\sigma$ is the process standard deviation. 
For normally distributed processes with one-sided specification limits, $C p u$ and $C p l$ indices are used to evaluate process capability where characteristics are smaller-to-better and larger-to-better, respectively, defined as follows.

$$
C_{p u}=\frac{U S L-\mu}{6 \sigma} \text { and } C_{p l}=\frac{\mu-L S L}{6 \sigma}
$$

From a practical perspective, the parameter $C_{p u}$ or $C_{p l}$ is typically unknown. Thus, we must use sample statistics to estimate it. For the estimations of the indices $C p u$ and $C p l$, we consider the following natural estimators.

$$
\hat{C}_{p u}=\frac{U S L-\bar{x}}{6 s} \text { and } \hat{C}_{p l}=\frac{\bar{x}-L S L}{6 s},
$$

where $\bar{x}$ is the sample mean and $s$ is the sample standard deviation. Chou and Owen [24] revealed that both $\hat{C}_{p u}$ and $\hat{C}_{p l}$ are noncentral $t$ distributions with $n-1$ degrees of freedom and noncentral parameter $\delta=3 \sqrt{n} C p u(\delta=3 \sqrt{n} C p l)$. For convenience of presentation, both $C p u$ and $C p l$ are denoted as $C_{S}$ in the subsequent sections.

\section{Proposed Sampling Plan}

In this section, we will describe the design of proposed sampling plan whose relevant contents are presented as follows.

\subsection{Procedure and Probability of Acceptance of the Repetitive Sampling Plan Based on the One-Sided PCI}

According to Sherman [18], the procedure of a repetitive sampling plan based on the one-sided PCI can be stated as follows.

Step 1: Determine the producer's risk $\alpha$, the consumer's risk $\beta$, and the corresponding process capability requirements $\left(C_{S}^{A Q L}\right.$ and $\left.C_{S}^{L T P D}\right)$.

Step 2: Obtain a random sample of size $n(>1)$ and compute $\hat{C}_{S}$.

Step 3: Formulate a decision on the lot as follows.

(1) Accept the lot if $\hat{C}_{s}>k_{a}$.

(2) Reject the lot if $\hat{C}_{s}<k_{r}$.

(3) Otherwise, repeat Step 2 by obtaining a new sample of size $n$.

Figure 1 presents a flow chart of a repetitive sampling plan based on the PCI.

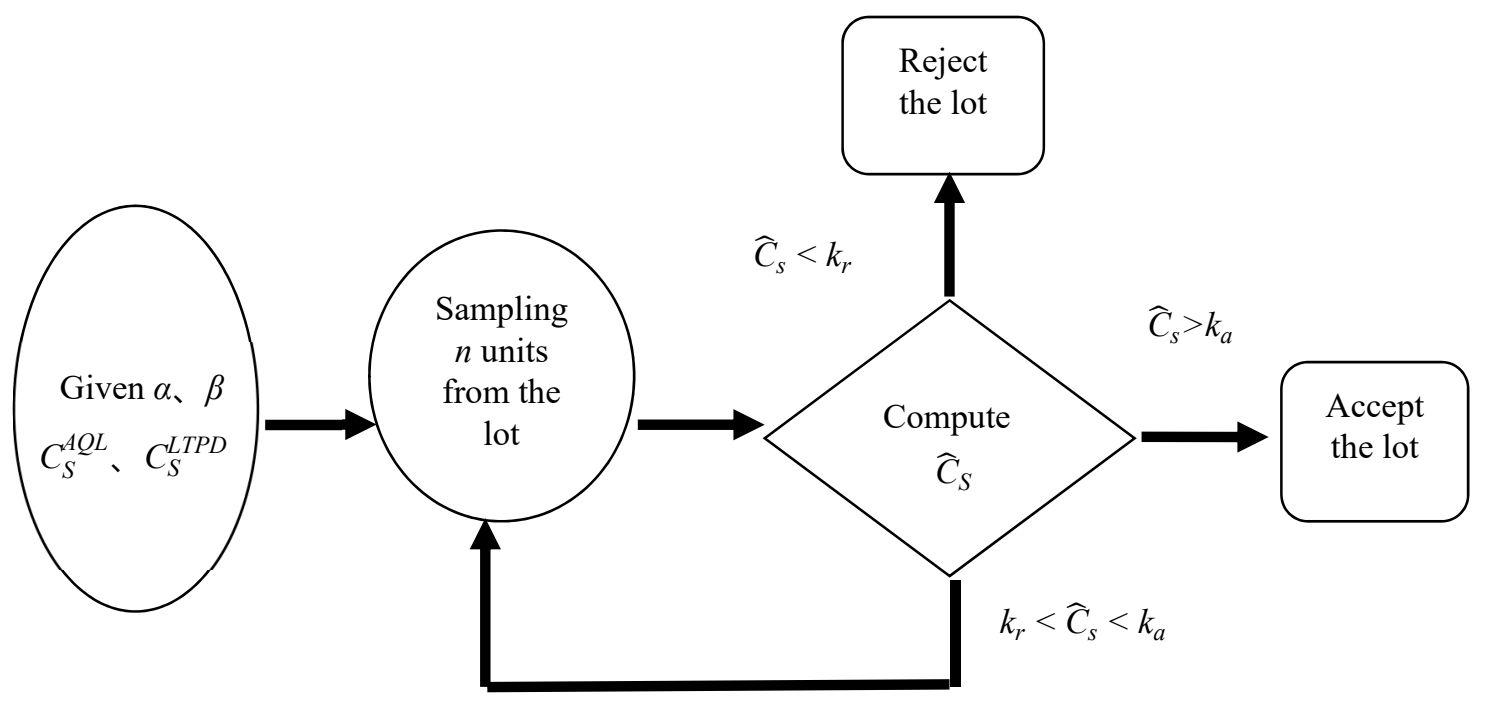

Figure 1. Flow chart of a repetitive sampling plan based on $C_{S}$. 
According to Yen et al. [8], the probability of acceptance of repetitive sampling based on one-sided PCI can be expressed as

$$
P_{R}=P_{a}+R P_{a}+R^{2} P_{a}+\cdots \cdots+R^{k} P_{a}+\cdots \cdots=\frac{P_{a}}{1-R}=\frac{P_{a}}{P_{a}+P_{r}}
$$

where $P_{a}=P\left(t_{n-1,3 \sqrt{n} C_{s}}>3 \sqrt{n} k_{a}\right), P_{r}=P\left(t_{n-1,3 \sqrt{n} C_{s}}<3 \sqrt{n} k_{r}\right), R=1-P_{a}-P_{r}$, and $t_{n-1,3 \sqrt{n} C_{s}}$ is a noncentral $\mathrm{t}$ distribution with $n-1$ degrees of freedom and noncentral parameter $3 \sqrt{n} C_{s}$.

\subsection{Derivation of Relevant Indices for the TQC Model}

With reference to Yen et al. [17], the quality cost model, TQC $=C_{i} A T I+C_{i f} D_{d}+C_{e f} D_{n}$, is adopted in this study. The TQC is a total quality cost including total inspection cost, total internal failure cost and total external failure cost while implementing the rectifying inspection, of which total inspection cost is equal to $C_{i} A T I$, total internal failure cost is equal to $C_{i f} D_{d}$ and total external failure cost is equal to $C_{e f} D_{n}$. Under repetitive sampling, the average total inspection (ATI), $D_{d}, D_{n}$, and average outgoing quality (AOQ) of rectifying inspection can be derived respectively as follows

$$
\begin{gathered}
A T I=\left[n P_{a}+2 n R P_{a}+3 n R^{2} P_{a}+\cdots \cdots\right]+N\left[1-P_{R}\right]=\frac{n P_{a}}{(1-R)^{2}}+\frac{N P_{r}}{P_{a}+P_{r}}=\frac{n P_{a}+N P_{r}\left(P_{a}+P_{r}\right)}{\left(P_{a}+P_{r}\right)^{2}} \\
D_{d}=\left[n P_{a}+2 n R P_{a}+3 n R^{2} P_{a}+\cdots \cdots\right] p+N\left[1-P_{R}\right] p=\frac{\left[n P_{a}+N P_{r}\left(P_{a}+P_{r}\right)\right] p}{\left(P_{a}+P_{r}\right)^{2}} \\
D_{n}=\left[P_{a}(N-n)+R P_{a}(N-2 n)+R^{2} P_{a}(N-3 n)+\cdots \cdots\right] p=\frac{P_{a}\left[N\left(P_{a}+P_{r}\right)-n\right] p}{\left(P_{a}+P_{r}\right)^{2}} \\
A O Q=\left[P_{a}(N-n)+R P_{a}(N-2 n)+R^{2} P_{a}(N-3 n)+\cdots \cdots\right] p=\frac{P_{a}\left[N\left(P_{a}+P_{r}\right)-n\right] p}{N\left(P_{a}+P_{r}\right)^{2}}
\end{gathered}
$$

\subsection{Mathematical Model of the Proposed Sampling Plan}

To evaluate the performance of the sampling plan, the operating characteristic (OC) curve is used to exhibit the discriminatory power. The OC curve plots the probability of accepting one lot versus the various proportion nonconforming. A typical OC curve is depicted in Figure 2. A supplier typically focuses on a specific product quality level, which is termed acceptable quality level (AQL), with a high probability $1-\alpha$ of accepting a lot. By contrast, a buyer typically focuses on a point at the other end of the OC curve that is termed lot tolerance percent defective (LTPD), with a low probability $\beta$ of accepting a lot. Thus, a well-designed sampling plan should provide a probability of at least $1-\alpha$ of accepting a lot if the product quality level achieves AQL and a probability of no more than $\beta$ if the level of the product quality is at the LTPD level. As such, the OC curve of the acceptance sampling plan passes through the two designated points (AQL, $1-\alpha)$ and (LTPD, $\beta$ ). To construct a PCI-based acceptance sampling plan, the corresponding two designated points of OC would be $\left(C_{S}^{A Q L}, 1-\alpha\right)$ and $\left(C_{S}^{L T P D}, \beta\right)$. 


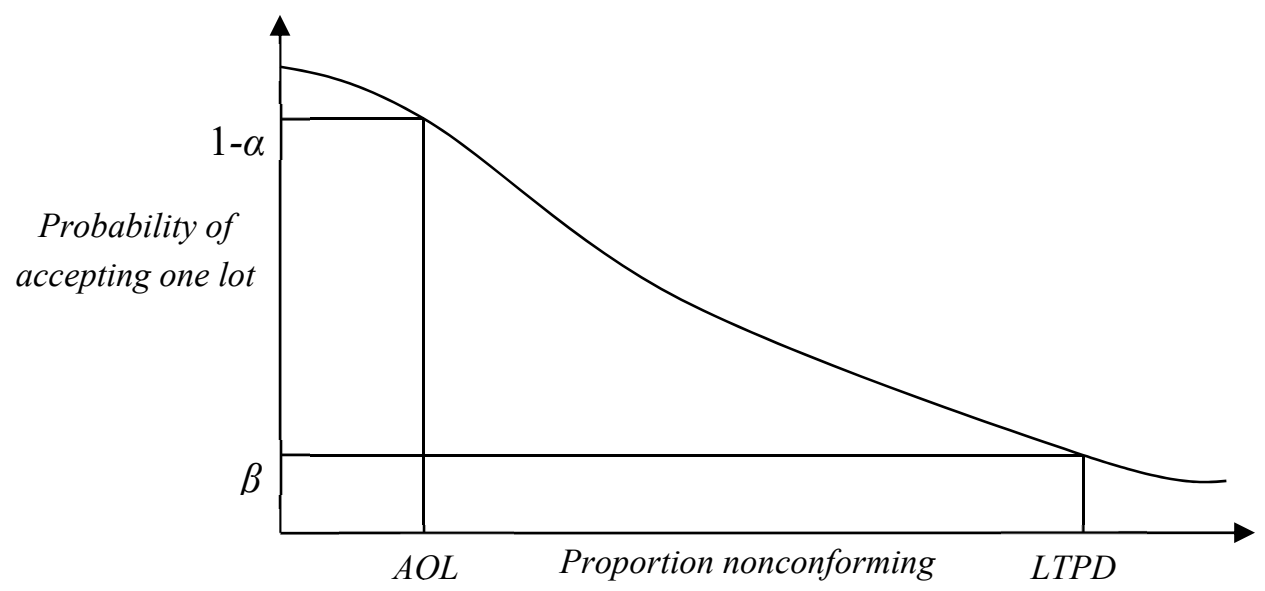

Figure 2. Conventional OC curve.

Consequently, the proposed sampling plan is developed based on the minimization of TQC while satisfying the two points of the OC curve. Therefore, a mathematical model of a repetitive rectifying sampling plan based on the one-sided PCI can be constructed as follows.

$$
\begin{gathered}
\text { Min TQC }=C_{i} A T I+C_{i f} D_{d}+C_{e f} D_{n} \\
\text { st. } P_{R}\left(C_{S}^{A Q L}\right) \geq 1-\alpha \\
P_{R}\left(C_{S}^{L T P D}\right) \leq \beta
\end{gathered}
$$

Based on Equations (1)-(4), the mathematical model can be expressed as follows.

$$
\begin{aligned}
& \text { Min TQC }=C_{i} \times \frac{n P_{a}+N P_{r}\left(P_{a}+P_{r}\right)}{\left(P_{a}+P_{r}\right)^{2}}+C_{i f} \times \frac{\left[n P_{a}+N P_{r}\left(P_{a}+P_{r}\right)\right] p}{\left(P_{a}+P_{r}\right)^{2}}+C_{e f} \frac{P_{a}\left[N\left(P_{a}+P_{r}\right)-n\right] p}{\left(P_{a}+P_{r}\right)^{2}}
\end{aligned}
$$

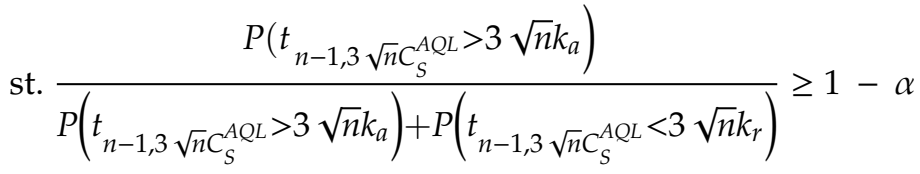

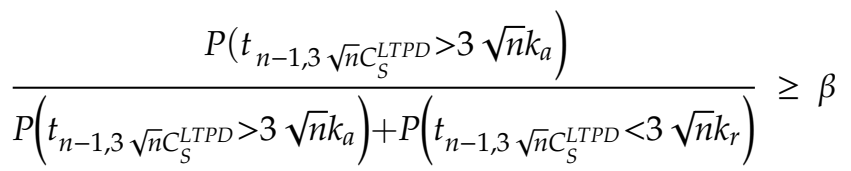

\section{Analysis and Discussion}

In this section, we provide numerical examples to illustrate the performance of the proposed sampling plan based on Equations (6)-(8) compared with that reported by Yen et al. [17].

The parameters of sampling plan and AOQ graph

For illustration, the relevant parameters of the numerical example are set at $\mathrm{N}=1000, C_{S}^{A Q L}=1.33$, $C_{S}^{L T P D}=1.0, C_{S}=1.165, C_{i}=10, C_{i f}=20$, and $C_{e f}=50$. The sample sizes, critical values, and TQC for both sampling plans with various combinations of $\alpha$ and $\beta$ are summarized in Table 1. From results in Table 1, we observe the following.

(i) The parameters of the two sampling plans are determined by consumer risk $\beta$ instead of producer risk $\alpha$.

(ii) For the two sampling plans, the sample size and the acceptance value both reveal a decreasing trend when consumer risk $\beta$ increases along with the fixed producer risk. Also, the sample size number of the repetitive rectifying sampling plan is significantly lower than that of the single rectifying sampling plan. 
(iii) When the producer risk $\alpha$ is fixed and the consumer risk $\beta$ increases, the TQC variables of both sampling plans decrease; the TQC of the repetitive sampling plan is significantly lower than that of the single sampling plan for all combinations.

Table 1. Parameters of the rectifying sampling plans and their corresponding total quality cost (TQC).

\begin{tabular}{|c|c|c|c|c|c|c|c|c|c|}
\hline \multirow[t]{2}{*}{$\alpha$} & \multirow[t]{2}{*}{$\beta$} & \multicolumn{3}{|c|}{$\begin{array}{l}\text { Single Sampling } \\
\text { (Yen et al. [17]) }\end{array}$} & \multicolumn{4}{|c|}{$\begin{array}{c}\text { Repetitive Sampling (The Proposed } \\
\text { Method) }\end{array}$} & \multirow{2}{*}{$\begin{array}{l}\text { Reduction on } \\
\text { TQC }\end{array}$} \\
\hline & & $n$ & $c$ & TQC & $n$ & $k_{a}$ & $k_{r}$ & TQC & \\
\hline \multirow{5}{*}{0.01} & 0.01 & 253 & 1.1262 & 4256.12 & 118 & 1.2101 & 1.0257 & 4040.99 & $5.05 \%$ \\
\hline & 0.025 & 223 & 1.1126 & 3588.23 & 96 & 1.2091 & 1.0034 & 3149.39 & $12.23 \%$ \\
\hline & 0.05 & 185 & 1.1036 & 3133.8 & 76 & 1.2121 & 0.9894 & 2582.92 & $17.58 \%$ \\
\hline & 0.075 & 169 & 1.0947 & 2809.77 & 93 & 1.1646 & 0.9987 & 2041.59 & $27.34 \%$ \\
\hline & 0.1 & 153 & 1.0886 & 2595.21 & 99 & 1.1446 & 0.9979 & 1822.37 & $29.78 \%$ \\
\hline \multirow{5}{*}{0.025} & 0.01 & 253 & 1.1262 & 4256.12 & 118 & 1.2101 & 1.0257 & 4040.99 & $5.05 \%$ \\
\hline & 0.025 & 223 & 1.1126 & 3588.23 & 96 & 1.2091 & 1.0034 & 3149.39 & $12.23 \%$ \\
\hline & 0.05 & 185 & 1.1036 & 3133.8 & 76 & 1.2121 & 0.9894 & 2582.92 & $17.58 \%$ \\
\hline & 0.075 & 169 & 1.0947 & 2809.77 & 93 & 1.1646 & 0.9987 & 2041.59 & $27.34 \%$ \\
\hline & 0.1 & 153 & 1.0886 & 2595.21 & 99 & 1.1446 & 0.9979 & 1822.37 & $29.78 \%$ \\
\hline \multirow{5}{*}{0.05} & 0.01 & 253 & 1.1262 & 4256.12 & 118 & 1.2101 & 1.0257 & 4040.99 & $5.05 \%$ \\
\hline & 0.025 & 223 & 1.1126 & 3588.23 & 96 & 1.2091 & 1.0034 & 3149.39 & $12.23 \%$ \\
\hline & 0.05 & 185 & 1.1036 & 3133.8 & 76 & 1.2121 & 0.9894 & 2582.92 & $17.58 \%$ \\
\hline & 0.075 & 169 & 1.0947 & 2809.77 & 93 & 1.1646 & 0.9987 & 2041.59 & $27.34 \%$ \\
\hline & 0.1 & 153 & 1.0886 & 2595.21 & 99 & 1.1446 & 0.9979 & 1822.37 & $29.78 \%$ \\
\hline \multirow{5}{*}{0.075} & 0.01 & 253 & 1.1262 & 4256.12 & 118 & 1.2101 & 1.0257 & 4040.99 & $5.05 \%$ \\
\hline & 0.025 & 223 & 1.1126 & 3588.23 & 96 & 1.2091 & 1.0034 & 3149.39 & $12.23 \%$ \\
\hline & 0.05 & 185 & 1.1036 & 3133.8 & 76 & 1.2121 & 0.9894 & 2582.92 & $17.58 \%$ \\
\hline & 0.075 & 169 & 1.0947 & 2809.77 & 93 & 1.1646 & 0.9987 & 2041.59 & $27.34 \%$ \\
\hline & 0.1 & 153 & 1.0886 & 2595.21 & 99 & 1.1446 & 0.9979 & 1822.37 & $29.78 \%$ \\
\hline \multirow{5}{*}{0.1} & 0.01 & 253 & 1.1262 & 4256.12 & 118 & 1.2101 & 1.0257 & 4040.99 & $5.05 \%$ \\
\hline & 0.025 & 223 & 1.1126 & 3588.23 & 96 & 1.2091 & 1.0034 & 3149.39 & $12.23 \%$ \\
\hline & 0.05 & 185 & 1.1036 & 3133.8 & 76 & 1.2121 & 0.9894 & 2582.92 & $17.58 \%$ \\
\hline & 0.075 & 169 & 1.0947 & 2809.77 & 93 & 1.1646 & 0.9987 & 2041.59 & $27.34 \%$ \\
\hline & 0.1 & 153 & 1.0886 & 2595.21 & 99 & 1.1446 & 0.9979 & 1822.37 & $29.78 \%$ \\
\hline
\end{tabular}

In addition, based on parameters in Table 1, we plot the graphs of AOQ for both of the sampling plans, as displayed in Figure 3. From Figure 3, we can see that the two sampling plans appear to be very similar, whereas the average outgoing quality limit (AOQL) of the repetitive sampling plan is slightly lower than that of the single sampling plan.

\subsection{Sensitivity Analysis}

To illustrate the sensitivity analysis, the values of the specific parameters are set at $\mathrm{N}=1000$, $C_{S}^{A Q L}=1.33, C_{S}^{L T P D}=1.0, \alpha=0.05$, and $\beta=0.1$. 
$\beta$

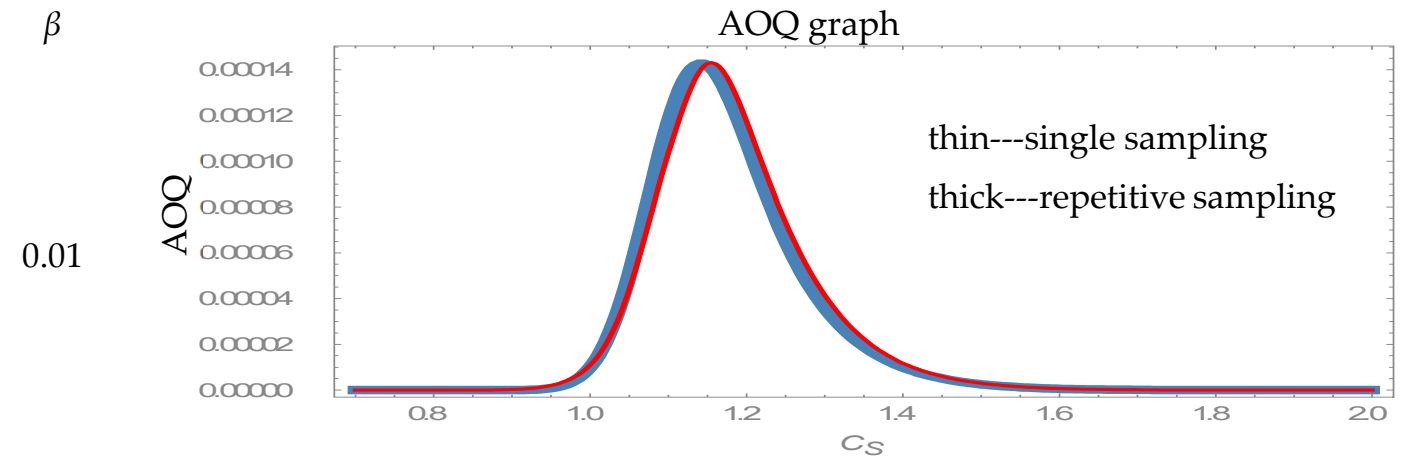

0.01
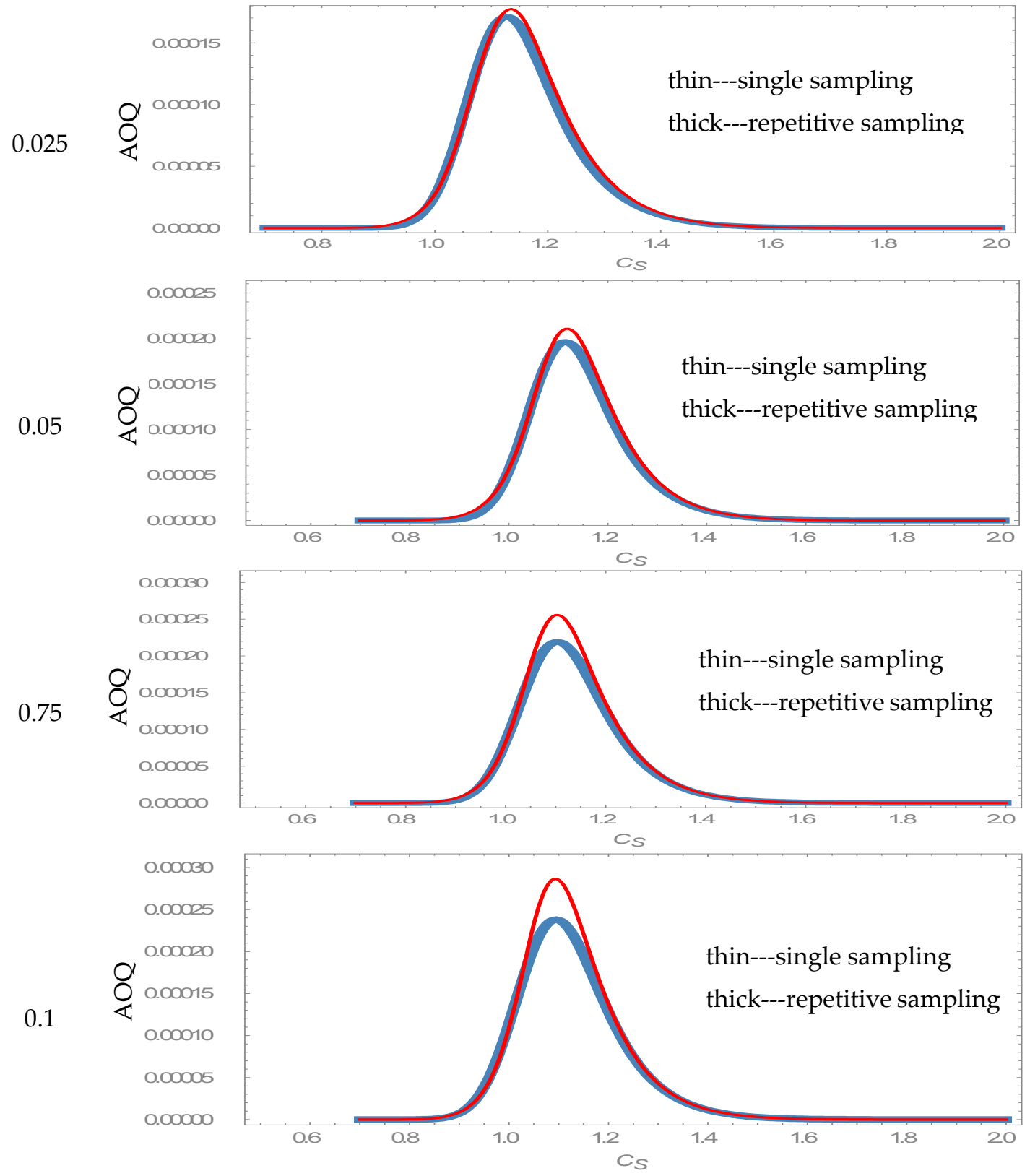

Figure 3. Graphs of average outgoing quality (AOQ) versus $C_{s}$ for the two rectifying sampling plans. 


\subsection{Sensitivity Analysis on TQC for Individual Parameters}

To investigate the behavior of the individual $C_{S}, C_{i}, C_{i f}$, and $C_{e f}$ against the TQC, sensitivity analysis is executed on TQC for individual parameters. Table 2 displays the sample size, critical values, and TQC of both sampling plans for $C_{S}=0.6(0.05) 1.6$ with the given $C_{i}=10, C_{i f}=20$, and $C_{e f}=50$. The results reveal that the sample size and critical values of the individual sampling plans are constant for all values of $C_{S}$, and the TQC of the proposed methods are significantly lower than those of the single sampling plan for all cases. Table 3 displays the sample size, critical values, and TQC of the two sampling plans for $C_{i}=10(10) 100, C_{i f}=10(10) 100$, and $C_{e f}=10(10) 100$ with the given $C_{S}=1.165$. The results reveal that the sample size and critical values of the individual sampling plans are constant regardless of changes in the values of $C_{i}, C_{i f}$, and $C_{e f}$, and the TQC of the proposed method is significantly lower than that of the single sampling plan for all cases. Figure 4 displays TQC vs. $C_{i}$, $C_{i f}$, and $C_{e f}$ based on results illustrated in Tables 2 and 3 . The outline reveals that the decline in TQC for the proposed method is more significant when the process capability is very effective (i.e., greater than 1.3) or very ineffective (i.e., less than 0.7). Moreover, the TQC seems to be primarily dependent on process capability and inspection cost, whereas internal failure cost and external failure cost have very little influence.

\subsection{Sensitivity Analysis on TQC for Simultaneous Changes in All Parameters}

To further investigate the extent of the individual parameters' effects on TQC, sensitivity analysis is implemented on TQC for simultaneous changes in $C_{S}, C_{i}, C_{i f}$, and $C_{e f}$. We establish three levels for each factor (i.e., $C_{S}, C_{i}, C_{i f}$, and $C_{e f}$ ) and use the orthogonal array $\mathrm{L}_{9}\left(3^{4}\right)$ to implement level average analysis. Table 4 exhibits the values of TQC for each combination of $C_{S}, C_{i}, C_{i f}$, and $C_{e f .}$, and the absolute values of the effects of each factor are presented in Table 5. From the results in Table 5, we conclude that $C_{S}$ has the strongest effect on TQC, followed by $C_{i}$, whereas $C_{i f}$ and $C_{e f}$ have the least effect on TQC.

Table 2. Sensitivity analysis of TQC for $C_{S}$.

\begin{tabular}{|c|c|c|c|c|c|c|c|c|c|c|c|}
\hline \multirow[t]{2}{*}{$C_{s}$} & \multirow[t]{2}{*}{$C_{i}$} & \multirow[t]{2}{*}{$C_{i f}$} & \multirow[t]{2}{*}{$C_{e f}$} & \multicolumn{3}{|c|}{$\begin{array}{l}\text { Single Sampling } \\
\text { (Yen et al. [17]) }\end{array}$} & \multicolumn{4}{|c|}{$\begin{array}{l}\text { Repetitive Sampling (The } \\
\text { Proposed Method) }\end{array}$} & \multirow[t]{2}{*}{$\begin{array}{l}\text { Reduction on } \\
\text { TQC }\end{array}$} \\
\hline & & & & $n$ & $c$ & TQC & $n$ & $k_{a}$ & $k_{r}$ & TQC & \\
\hline 0.6 & & & & 153 & 1.0886 & $12,240.3$ & 99 & 1.1446 & 0.9979 & $10,718.6$ & $12.43 \%$ \\
\hline 0.65 & & & & 153 & 1.0886 & $11,595.4$ & 99 & 1.1446 & 0.9979 & $10,511.8$ & $9.35 \%$ \\
\hline 0.7 & & & & 153 & 1.0886 & $11,113.8$ & 99 & 1.1446 & 0.9979 & $10,357.3$ & $6.81 \%$ \\
\hline 0.75 & & & & 153 & 1.0886 & $10,762.2$ & 99 & 1.1446 & 0.9979 & $10,244.5$ & $4.81 \%$ \\
\hline 0.8 & & & & 153 & 1.0886 & $10,511.1$ & 99 & 1.1446 & 0.9979 & $10,163.7$ & $3.31 \%$ \\
\hline 0.85 & & & & 153 & 1.0886 & $10,334.3$ & 99 & 1.1446 & 0.9979 & $10,104.8$ & $2.22 \%$ \\
\hline 0.9 & & & & 153 & 1.0886 & $10,194.1$ & 99 & 1.1446 & 0.9979 & $10,044.6$ & $1.47 \%$ \\
\hline 0.95 & & & & 153 & 1.0886 & 9957.21 & 99 & 1.1446 & 0.9979 & 9884.05 & $0.73 \%$ \\
\hline 1 & & & & 153 & 1.0886 & 9230.03 & 99 & 1.1446 & 0.9979 & 9219.02 & $0.12 \%$ \\
\hline 1.05 & & & & 153 & 1.0886 & 7530.42 & 99 & 1.1446 & 0.9979 & 7174.3 & $4.73 \%$ \\
\hline 1.1 & & & & 153 & 1.0886 & 5103.36 & 99 & 1.1446 & 0.9979 & 4116.83 & $19.33 \%$ \\
\hline 1.15 & 10 & 20 & 50 & 153 & 1.0886 & 3024.57 & 99 & 1.1446 & 0.9979 & 2137.05 & $29.34 \%$ \\
\hline 1.2 & & & & 153 & 1.0886 & 1947.55 & 99 & 1.1446 & 0.9979 & 1384.32 & $28.92 \%$ \\
\hline 1.25 & & & & 153 & 1.0886 & 1606.06 & 99 & 1.1446 & 0.9979 & 1124.35 & $29.99 \%$ \\
\hline 1.3 & & & & 153 & 1.0886 & 1539.02 & 99 & 1.1446 & 0.9979 & 1032.12 & $32.94 \%$ \\
\hline 1.35 & & & & 153 & 1.0886 & 1530.75 & 99 & 1.1446 & 0.9979 & 1001.43 & $34.58 \%$ \\
\hline 1.4 & & & & 153 & 1.0886 & 1530.07 & 99 & 1.1446 & 0.9979 & 992.722 & $35.12 \%$ \\
\hline 1.45 & & & & 153 & 1.0886 & 1530.02 & 99 & 1.1446 & 0.9979 & 990.656 & $35.25 \%$ \\
\hline 1.5 & & & & 153 & 1.0886 & 1530.01 & 99 & 1.1446 & 0.9979 & 990.202 & $35.28 \%$ \\
\hline 1.55 & & & & 153 & 1.0886 & 1530.01 & 99 & 1.1446 & 0.9979 & 990.082 & $35.29 \%$ \\
\hline 1.6 & & & & 153 & 1.0886 & 1530 & 99 & 1.1446 & 0.9979 & 990.038 & $35.29 \%$ \\
\hline 1.8 & & & & 153 & 1.0886 & 1530 & 99 & 1.1446 & 0.9979 & 990.002 & $35.29 \%$ \\
\hline 2 & & & & 153 & 1.0886 & 1530 & 99 & 1.1446 & 0.9979 & 990 & $35.29 \%$ \\
\hline
\end{tabular}


Table 3. Sensitivity analysis of TQC for $C_{i}, C_{i f}$, and $C e f$.

\begin{tabular}{|c|c|c|c|c|c|c|c|c|c|c|c|}
\hline \multirow[t]{2}{*}{$C_{s}$} & \multirow[t]{2}{*}{$C_{i}$} & \multirow[t]{2}{*}{$C_{i f}$} & \multirow[t]{2}{*}{$C_{e f}$} & \multicolumn{3}{|c|}{$\begin{array}{l}\text { Single Sampling } \\
\text { (Yen et al. [17]) }\end{array}$} & \multicolumn{4}{|c|}{$\begin{array}{l}\text { Repetitive Sampling (The } \\
\text { Proposed Method) }\end{array}$} & \multirow{2}{*}{$\begin{array}{l}\text { Reduction on } \\
\text { TQC }\end{array}$} \\
\hline & & & & $n$ & $c$ & TQC & $n$ & $k_{a}$ & $k_{r}$ & TQC & \\
\hline \multirow{10}{*}{1.165} & 10 & \multirow{10}{*}{20} & \multirow{10}{*}{50} & 153 & 1.0886 & 2595.21 & 99 & 1.1446 & 0.9979 & 1822.37 & $29.78 \%$ \\
\hline & 20 & & & 153 & 1.0886 & 5187.92 & 99 & 1.1446 & 0.9979 & 3634.17 & $29.95 \%$ \\
\hline & 30 & & & 153 & 1.0886 & 7780.64 & 99 & 1.1446 & 0.9979 & 5445.98 & $30.01 \%$ \\
\hline & 40 & & & 153 & 1.0886 & $10,373.4$ & 99 & 1.1446 & 0.9979 & 7257.79 & $30.03 \%$ \\
\hline & 50 & & & 153 & 1.0886 & $12,966.1$ & 99 & 1.1446 & 0.9979 & 9069.59 & $30.05 \%$ \\
\hline & 60 & & & 153 & 1.0886 & $15,558.8$ & 99 & 1.1446 & 0.9979 & $10,881.4$ & $30.06 \%$ \\
\hline & 70 & & & 153 & 1.0886 & $18,151.5$ & 99 & 1.1446 & 0.9979 & $12,693.2$ & $30.07 \%$ \\
\hline & 80 & & & 153 & 1.0886 & $20,744.2$ & 99 & 1.1446 & 0.9979 & 14,505 & $30.08 \%$ \\
\hline & 90 & & & 153 & 1.0886 & 23,337 & 99 & 1.1446 & 0.9979 & $16,316.8$ & $30.08 \%$ \\
\hline & 100 & & & 153 & 1.0886 & $25,929.7$ & 99 & 1.1446 & 0.9979 & $18,128.6$ & $30.09 \%$ \\
\hline \multirow{10}{*}{1.165} & \multirow{10}{*}{10} & 10 & \multirow{10}{*}{50} & 153 & 1.0886 & 2594.59 & 99 & 1.1446 & 0.9979 & 1821.94 & $29.78 \%$ \\
\hline & & 20 & & 153 & 1.0886 & 2595.21 & 99 & 1.1446 & 0.9979 & 1822.37 & $29.78 \%$ \\
\hline & & 30 & & 153 & 1.0886 & 2595.82 & 99 & 1.1446 & 0.9979 & 1822.8 & $29.78 \%$ \\
\hline & & 40 & & 153 & 1.0886 & 2596.44 & 99 & 1.1446 & 0.9979 & 1823.23 & $29.78 \%$ \\
\hline & & 50 & & 153 & 1.0886 & 2597.05 & 99 & 1.1446 & 0.9979 & 1823.66 & $29.78 \%$ \\
\hline & & 60 & & 153 & 1.0886 & 2597.66 & 99 & 1.1446 & 0.9979 & 1824.09 & $29.78 \%$ \\
\hline & & 70 & & 153 & 1.0886 & 2598.28 & 99 & 1.1446 & 0.9979 & 1824.52 & $29.78 \%$ \\
\hline & & 80 & & 153 & 1.0886 & 2598.89 & 99 & 1.1446 & 0.9979 & 1824.95 & $29.78 \%$ \\
\hline & & 90 & & 153 & 1.0886 & 2599.51 & 99 & 1.1446 & 0.9979 & 1825.38 & $29.78 \%$ \\
\hline & & 100 & & 153 & 1.0886 & 2600.12 & 99 & 1.1446 & 0.9979 & 1825.8 & $29.78 \%$ \\
\hline \multirow{10}{*}{1.165} & \multirow{10}{*}{10} & \multirow{10}{*}{20} & 10 & 153 & 1.0886 & 2594.2 & 99 & 1.1446 & 0.9979 & 1814.61 & $30.05 \%$ \\
\hline & & & 20 & 153 & 1.0886 & 2594.45 & 99 & 1.1446 & 0.9979 & 1816.55 & $29.98 \%$ \\
\hline & & & 30 & 153 & 1.0886 & 2594.7 & 99 & 1.1446 & 0.9979 & 1818.49 & $29.92 \%$ \\
\hline & & & 40 & 153 & 1.0886 & 2594.95 & 99 & 1.1446 & 0.9979 & 1820.43 & $29.85 \%$ \\
\hline & & & 50 & 153 & 1.0886 & 2595.21 & 99 & 1.1446 & 0.9979 & 1822.37 & $29.78 \%$ \\
\hline & & & 60 & 153 & 1.0886 & 2595.46 & 99 & 1.1446 & 0.9979 & 1824.31 & $29.71 \%$ \\
\hline & & & 70 & 153 & 1.0886 & 2595.71 & 99 & 1.1446 & 0.9979 & 1826.25 & $29.64 \%$ \\
\hline & & & 80 & 153 & 1.0886 & 2595.96 & 99 & 1.1446 & 0.9979 & 1828.19 & $29.58 \%$ \\
\hline & & & 90 & 153 & 1.0886 & 2596.21 & 99 & 1.1446 & 0.9979 & 1830.13 & $29.51 \%$ \\
\hline & & & 100 & 153 & 1.0886 & 2596.47 & 99 & 1.1446 & 0.9979 & 1832.07 & $29.44 \%$ \\
\hline
\end{tabular}

Table 4. Sensitivity analysis for simultaneous changes in parameters.

\begin{tabular}{|c|c|c|c|c|c|c|c|c|c|c|c|}
\hline \multirow[t]{2}{*}{$L_{9}\left(3^{4}\right)$} & \multirow[b]{2}{*}{$C_{S}$} & \multirow[b]{2}{*}{$C_{i}$} & \multirow[b]{2}{*}{$C_{i f}$} & \multirow[b]{2}{*}{$C_{e f}$} & \multicolumn{3}{|c|}{$\begin{array}{l}\text { Single Sampling } \\
\text { (Yen et al. [17]) }\end{array}$} & \multicolumn{4}{|c|}{$\begin{array}{l}\text { Repetitive Sampling (The } \\
\text { Proposed Method) }\end{array}$} \\
\hline & & & & & $n$ & $c$ & TQC & $n$ & $k_{a}$ & $k_{r}$ & TQC \\
\hline 1 & 0.9 & 10 & 40 & 70 & 153 & 1.0886 & $10,321.9$ & 99 & 1.1446 & 0.9979 & $10,113.9$ \\
\hline 2 & 0.9 & 20 & 50 & 80 & 153 & 1.0886 & $20,364.2$ & 99 & 1.1446 & 0.9979 & $20,123.6$ \\
\hline 3 & 0.9 & 30 & 60 & 90 & 153 & 1.0886 & $30,406.6$ & 99 & 1.1446 & 0.9979 & $30,133.3$ \\
\hline 4 & 1.2 & 10 & 50 & 90 & 153 & 1.0886 & 1948.74 & 99 & 1.1446 & 0.9979 & 1390.47 \\
\hline 5 & 1.2 & 20 & 60 & 70 & 153 & 1.0886 & 3895.52 & 99 & 1.1446 & 0.9979 & 2764.97 \\
\hline 6 & 1.2 & 30 & 40 & 80 & 153 & 1.0886 & 5841.57 & 99 & 1.1446 & 0.9979 & 4142.93 \\
\hline 7 & 1.5 & 10 & 60 & 80 & 153 & 1.0886 & 1530.03 & 99 & 1.1446 & 0.9979 & 990.307 \\
\hline 8 & 1.5 & 20 & 40 & 90 & 153 & 1.0886 & 3060.02 & 99 & 1.1446 & 0.9979 & 1980.37 \\
\hline 9 & 1.5 & 30 & 50 & 70 & 153 & 1.0886 & 4590.03 & 99 & 1.1446 & 0.9979 & 2970.36 \\
\hline
\end{tabular}

Table 5. Influence of parameters.

\begin{tabular}{cccccccccc}
\hline & \multicolumn{4}{c}{ Single Sampling (Yen et al. [17]) } & \multicolumn{3}{c}{ Repetitive Sampling (The Proposed Method) } \\
\cline { 2 - 9 } & \multicolumn{1}{c}{$C_{S}$} & $C_{i}$ & $C_{i f}$ & $C_{e f}$ & $C_{S}$ & $C_{i}$ & $C_{i f}$ & $C_{e f}$ \\
\hline effect & $17,304.207$ & 9012.51 & 5536.22 & 5535.97 & $18,143.254$ & 8250.6377 & 5883.7923 & 5884.97 \\
\hline
\end{tabular}



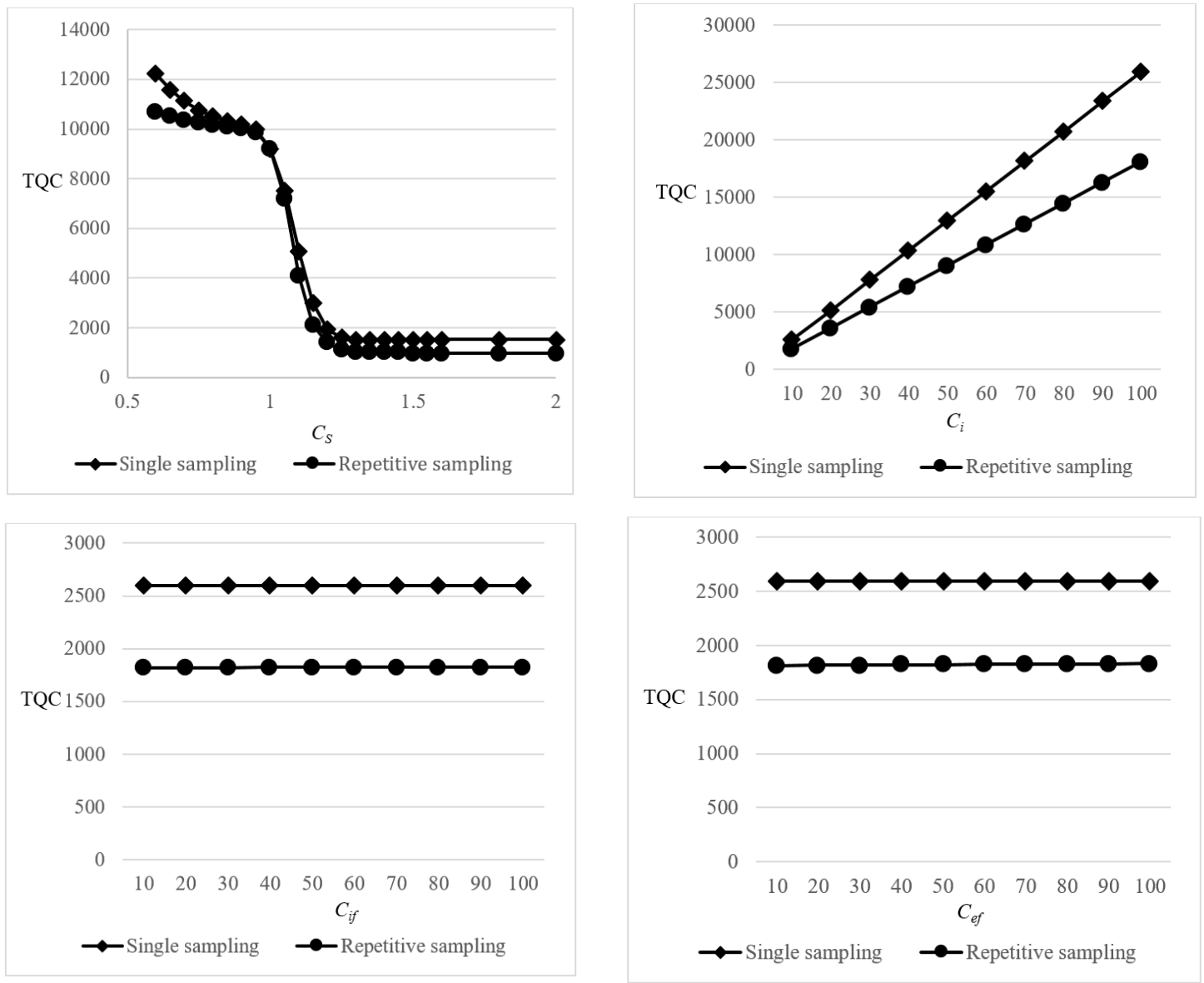

Figure 4. Sensitivity analysis graphs for $C_{S}, C_{i}, C_{i f}$, and $C_{e f}$.

\section{Conclusions}

In this paper, we propose a repetitive rectifying sampling plan based on the one-sided PCI by extending previous research on the single rectifying sampling plan [17]. Our proposed repetitive rectifying sampling plan minimizes $T Q C$, including inspection cost, internal failure cost, and external failure cost. A thorough comparison with the single rectifying sampling plan is implemented to confirm the performance of the proposed sampling plan. The results of the parameters of the two sampling plans reveal that sample size and critical values seem to be only determined by consumer risk rather than producer risk, quality level, or relevant quality costs. For various risk combinations under certain parameter conditions, the TQC of the proposed sampling plan is significantly lower than that of the single rectifying sampling plan. Specifically, the TQC of the proposed sampling plan is extremely low relative to that of the single rectifying sampling plan when the process capability is very good or when it is very bad. Furthermore, the AOQL of the proposed sampling plan is lower than that of the single rectifying sampling plan despite the two sampling plans' apparently similar AOQ. Based on the results of the sensitivity analysis, we observe that $C_{S}$ has the strongest effect on TQC, followed by $C_{i}$, whereas $C_{i f}$ and $C_{e f}$ exhibit the least influence on TQC. Overall, we conclude that the proposed sampling plan exhibits superior performance to that of Yen et al. [17]. Therefore, the proposed sampling plan is recommended when a rectifying inspection must be executed. It is noted that the proposed methodology is applied in the quality characteristic of interest that follows normal distribution and has one-sided specifications.

Author Contributions: Conceptualization, C.-H.Y; Data curation, Y.-R.S.; Formal analysis, K.-H.L.; Investigation, K.-H.L. and S.-H.L.; Methodology, C.-H.Y; Project administration, C.-C.L.; Resources, S.-H.L.; Software, Y.-R.S.; 
Supervision, C.-C.L.; Writing—original draft, C.-H.Y. All authors have read and agreed to the published version of the manuscript.

Funding: This research received no external funding.

Acknowledgments: The authors appreciate the support of Ministry of Sciences and Technology of Taiwan.

Conflicts of Interest: The authors declare no conflict of interest.

\section{Nomenclature}

TQC total quality cost per lot

ATI average total inspection

$A O Q \quad$ average outgoing quality

$C_{i} \quad$ inspection cost per unit

$C_{\text {if }} \quad$ internal failure cost per unit

$C_{e f} \quad$ external failure cost per item

N lot size

$c \quad$ acceptance value of single sampling

$k_{r} \quad$ rejection value of repetitive sampling

$C_{S}^{L T P D} \quad$ lot tolerance percent defective of one-sided PCI

$D_{d} \quad$ defective units detected

$D_{n} \quad$ defective units undetected

$P_{a} \quad$ probability of accepting one lot for each sampling

$P_{r} \quad$ probability of rejecting one lot for each sampling

$R \quad$ probability of resampling after each sampling

$P_{R} \quad$ probability of acceptance of repetitive sampling plan

$n \quad$ sample size

$k_{a} \quad$ acceptance value of repetitive sampling

$C_{S}^{A Q L} \quad$ acceptable quality level of one-sided PCI

\section{References}

1. Schilling, E.G. Acceptance Sampling in Quality Control; Marcel Dekker Inc.: New York, NY, USA, 1982.

2. Duncan, A.J. Quality Control and Industrial Statistics, 5th ed.; Irwin: Huntersville, NC, USA, 1986.

3. Montgomery, D.C. Introduction to Statistical Quality Control, 5th ed.; John Wiley: New York, NY, USA, 2005.

4. Yen, C.-H.; Aslam, M.; Jun, C.-H. A lot inspection sampling plan based on EWMA yield index. Int. J. Adv. Manuf. Technol. 2014, 75, 861-868. [CrossRef]

5. Wu, C.-W.; Liu, S.-W. Developing a sampling plan by variables inspection for controlling lot fraction of defectives. Appl. Math. Model. 2014, 38, 2303-2310. [CrossRef]

6. Aslam, M.; Yen, C.-H.; Chang, C.-H.; Jun, C.-H. Multiple dependent state variable sampling plans with process loss consideration. Int. J. Adv. Manuf. Technol. 2014, 71, 1337-1343. [CrossRef]

7. Wu, C.-W.; Shu, M.-H.; Nugroho, A.A.; Kurniati, N. A flexible process-capability-qualified resubmission-allowed acceptance sampling scheme. Comput. Ind. Eng. 2015, 80, 62-71. [CrossRef]

8. Yen, C.H.; Chang, C.H.; Aslam, M. Repetitive variable acceptance sampling plan for one-sided specification. J. Stat. Comput. Simul. 2015, 85, 1102-1116. [CrossRef]

9. Lee, A.H.; Wu, C.W.; Chen, Y.W. A modified variables repetitive group sampling plan with the consideration of preceding lots information. Ann. Oper. Res. 2016, 238, 355-373. [CrossRef]

10. Fallah-Nezhad, M.S.; Seifi, S. Repetitive group sampling plan based on the process capability index for the lot acceptance problem. J. Stat. Comput. Simul. 2017, 87, 29-41. [CrossRef]

11. Wu, C.W.; Wang, Z.H. Developing a variables multiple dependent state sampling plan with simultaneous consideration of process yield and quality loss. Int. J. Prod. Res. 2017, 55, 2351-2364. [CrossRef]

12. Yen, C.H.; Chang, C.H.; Aslam, M.; Jun, C.H. Multiple Dependent State Repetitive Sampling Plans for One-Sided process capability indices. Commun. Stat. Theory Methods 2018, 47, 1403-1412. [CrossRef]

13. Aslam, M.; Balamurali, S.; Jun, C.-H. A new multiple dependent state sampling plan based on the process capability index. Commun. Stat. Simul. Comput. 2019. [CrossRef]

14. Feigenbaum, A.V. Total Quality Control, 3rd ed.; McGraw-Hill: New York, NY, USA, 1983. 
15. Juran, J.M. The Quality Trilogy. Qual. Prog. 1986, 19, 19-24.

16. Shank, J.K.; Govindarajan, V. Strategic Cost Management: The New Tool of Competitive Advantage; Free Press: New York, NY, USA, 1993.

17. Yen, C.-H.; Ma, H.; Yeh, C.-H.; Chang, C.-H. The economic design of variable acceptance sampling plan with rectifying inspection. Kybernetes 2015, 44, 440-450. [CrossRef]

18. Sherman, R.E. Design and evaluation of a repetitive group sampling plan. Technometrics 1965, 7, 11-21. [CrossRef]

19. Wortham, A.W.; Baker, R.C. Multiple deferred state sampling inspection. Int. J. Prod. Res. 1976, 14, 719-731. [CrossRef]

20. Aslam, M.; Yen, C.-H.; Chang, C.-H.; Jun, C.-H. Multiple states repetitive group sampling plans with process loss consideration. Appl. Math. Model. 2013, 37, 9063-9075. [CrossRef]

21. Juran, J.M. Quality Control Handbook, 3rd ed.; McGraw-Hill: New York, NY, USA, 1974.

22. Kane, V.E. Process Capability Indices. J. Qual. Technol. 1986, 18, 41-52. [CrossRef]

23. Chan, L.K.; Cheng, S.W.; Spiring, F.A. A New Measure of Process Capability: Cpm. J. Qual. Technol. 1988, 20, 162-175. [CrossRef]

24. Chou, Y.-M.; Owen, D. On the distributions of the estimated process capability indices. Commun. Stat. Theory Methods 1989, 18, 4549-4560. [CrossRef]

(C) 2020 by the authors. Licensee MDPI, Basel, Switzerland. This article is an open access article distributed under the terms and conditions of the Creative Commons Attribution (CC BY) license (http://creativecommons.org/licenses/by/4.0/). 